\title{
LA MODA COME LINGUAGGIO: INTERVISTA A GIUSY MENONCELLO ${ }^{1}$
}

Maria Aparecida Cardoso Santos ${ }^{2}$

Maria Franca Zuccarello ${ }^{3}$

$\mathcal{N}$ ata a Treviso, dopo aver seguito una formazione frequentando scuole per stilisti e scuole ad indirizzo artistico, dal 1983 inizia una professione nel settore abbigliamento maturando competenze nell'ambito della produzione e della creazione del prodotto.

Le esperienze di progettazione modellistica si sono ampliate poi alla progettazione stilistica, seguendo la realizzazione del prodotto nell'azienda.

Dal 1995 ad oggi si affianca alla progettazione un'attività didattica per vari enti e scuole dell'area trevigiana con le materie di: Stilismo / Disegno, Modellistica, Modellistica applicata al Cad e Confezione Industriale.

Attualmente esercita consulenze stilistiche/grafiche e modellistiche occupandosi della progettazione e realizzazione del campionario, seguendo anche la modellistica del capo.

Vista la formazione di design, la parte grafica che correda il campionario viene approfondita con un'attività di ricerca, studiando i vari metodi di realizzazione per tutti i tipi di tessuto.

\section{RIU: Com'è nata la passione per l'abbigliamento?}

G.Menoncello: La passione per la moda è nata quand'ero ragazzina verso i 10 anni, io avevo una zia sarta e, mi appassionava molto vedere quello che lei riusciva fare anche

\footnotetext{
${ }^{1}$ Consulente stilistica / grafica per alcune aziende d'abbigliamento e dall'insegnamento di stilismo e modellistica presso istituti superiori. E-mail: g_menoncello@virgilio.it. Homepage: www.gyon.it

${ }^{2}$ Professora Adjunta da Universidade do Estado do Rio de Janeiro/ILE/LNEO/Setor de Italiano (e-mail :aparecida.cardoso@yahoo.it).

${ }_{3}^{3}$ Professora Adjunta da Universidade do Estado do Rio de Janeiro/ILE/LNEO/Setor de Italiano (e-mail: mafrancazuccarello@superig.com.br).
} 
con un banale pezzo di stoffa la sua creatività e, spesso passavo molto tempo con lei cercando di imitarla, facendogli molte domande sul suo lavoro, cercando di realizzare delle piccole cose anch'io, o dando qualche punto a mano (sottopunti) sui capi che lei stava realizzando.

RIU: Abbiamo visto dal suo curriculum che la moda stilismo è per Lei una manifestazione di arte. È vero?

G.Menoncello: Si, penso che chi come me ha una passione l'arte e il costume, la moda e lo stilismo possano essere una manifestazione d'arte. Ritengo sia molto interessante rivedere alcune opere del passato, anche di grandi artisti rinascimentali o semplicemente alcuni elementi importanti di un quadro e, proponendoli in una chiave più moderna, riproporle mescolando tecniche industriali e artigianali che rendono queste immagini portabili, magari come stampa per una t-shirt ; oppure come disegno di base per la realizzazione di un tessuto a metraggio.

RIU: Dal suo sito ci appare che Lei fa una nuova interpretazione di disegni di tappezzerie antiche. Potremmo quindi dire che Lei, con la sua arte, sta trasformando un arazzo in un quadro dell'attualità?

G.Menoncello: Con il mio modo di fare arte e con quelle che sono le mie conoscenze pittoriche e di tecniche di stampa cerco interpretare alcuni disegni antichi rendendoli più nuovi ed attuali facendoli diventare dei quadri, spesso realizzando questi su delle basi di tessuti per abbigliamento (tipo lino, seta, raso di seta). A volte questi quadri hanno avuto delle evoluzioni, le immagini realizzate le ho poi utilizzate come degli inserimenti di stampe all'interno di alcune collezioni.

RIU: Si può dire che la moda ha il suo proprio linguaggio, la sua propria sintassi in termine di organizzazione. In che modo, per esempio, gli studi linguistici "stricto sensu" potrebbero coniugarsi allo studio ed allo sviluppo dei contesti stilistici? 
G.Menoncello: Certamente la moda ha un suo linguaggio: la moda è un movimento a sfondo sociale che ha sempre caratterizzato un modo ed uno stile di vita, infatti il modo di abbigliarsi è sempre dettato dal contesto in cui una persona vive. Credo sia molto importante studiare i costumi nei secoli, soprattutto dal ' 900 ai giorni nostri, cioè i grandi movimenti sociali, a volte nati anche come movimento di protesta ma, che hanno portato dei cambiamenti stilistici e di stili di vita.

RIU: In che maniera e misura Lei pensa che il linguaggio della moda possa interessare ed essere interessante per lo studente d'italiano all'estero?

G.Menoncello: Credo possa diventare interessante studiando alcuni grandi del costume da Chanel a Dior, arrivando ai nostri giorni Armani, Versace ed altri, capire quali sono state le loro innovazioni non solo stilistiche ma anche modellistiche di costruzione del capo, il new look di Dior le giacche di Armani. Le innovazioni merceologiche, il tessuto Chanel, la maglia metallo di Versace ed altro: affianco a queste innovazioni di metodo si è inserito anche un nuovo modo di esprimersi arricchendo il vocabolario della moda.

\section{RIU: Dal 1995 ad oggi Lei si dedica all'attività didattica nell'ambito della moda.} Cosa potrebbe dire agli studenti d'italiano all'estero a riguardo dell'importanza dello studio della lingua?

G.Menoncello: Una corretta proprietà di linguaggio data da una conoscenza stilistica, modellistica e merceologica permette una maggiore professionalità ed un miglior modo di interagire con il cliente soprattutto con i produttori di abbigliamento arginando cosi la possibilità di errore, diminuendo gli sprechi e la perdite di tempo e realizzando con maggiore sicurezza ciò che si vuole.

RIU: Ci sembra che negli ultimi anni la moda brasiliana stia prendendo piede anche in Italia. Cosa ne pensa?

G.Menoncello: La moda, come l'arte, è contaminazione, siamo nell'era della globalizzazione e della comunicazione, pertanto anche i costumi cambiano, tutto ciò può essere un arricchimento per un nuovo modo di vestire. 
RIU: Potremmo dire che quella brasiliana sia una nuova maniera di far moda, volta a far diventare più leggeri e versatili indumenti in genere considerati abbastanza seri? Secondo Lei il clima tropicale in relazione a quello italiano può contribuire a questo senso di leggerezza nella moda?

G.Menoncello: Penso che possa fornire una nuova proposta, un'alternativa ad alcuni modi di vestire più tradizionali facenti parte della cultura italiana e che mescolati con saggezza a capi più tradizionali possano essere anche un nuovo trend.

RIU: La realizzazione di collezioni, la consulenza stilistica/grafica e modellistica, ed infine la sua attività didattica, la fanno sentire un'unica professionista o varie?

G.Menoncello: Mi fanno sentire una professionista "creativa" che cerca di esprimersi in vari modi, perché la creatività non si esprime in un'unica forma ma in molteplici, e una persona che cerca di trasmettere il proprio sapere ad altri attraverso la didattica.

Treviso 13 Settembre 2011 